\title{
The Recording and Quantification of Event-Related Potentials: I. Stimulus Presentation and Data Acquisition
}

\author{
Paniz Tavakoli, a, Kenneth Campbell a \\ a School of Psychology, University of Ottawa
}

\begin{abstract}
Event-related potentials (ERPs) are the changes in the ongoing electrical activity of the brain (the EEG) that are elicited by either an external physical stimulus or an internal psychological "event". This article provides a tutorial review of the methods used for the collection of ERP data. Because ERPs are influenced by both stimulus parameters and the mental state of the subject (what the subject is "doing"), precise control over how the stimulus is presented and how the subject's response is monitored must be described. ERPs are generally recorded from electrodes placed on the scalp. How the electrodes are placed (the montage) and the choice of the reference to which the electrical activity of the scalp are compared will have a large influence on the results. Electrodes will also pick up extraneous artifact or "noise". Methods to reduce this noise are described. ERPs provide high temporal resolution of the extent of information processing allowing researchers to access to both sensory and cognitive processes involved in complex decision-making.
\end{abstract}

Keywords " Event-related potentials, data acquisition, stimulus parameters, EEG, noise, artifact

Đkcampbel@uottawa.ca

\section{Introduction}

Event-related potentials (ERPs) are becoming an increasingly important tool in the study of cognitive neuroscience. The novice can however be easily overwhelmed with the need to learn a vast array of techniques. This article provides a tutorial review of stimulus presentation and acquisition techniques for an ERP study. An ERP is a change in the ongoing electrical activity of the brain associated with either an external physical stimulus or an internal psychological "event". The amplitude of the ERP that occurs to the stimulus or psychological event is typically very small and is embedded in the much larger amplitude of the ongoing electroencephalogram (EEG). Thus, what is recorded is a mixture of a true "signal" (the response) occurring in the ongoing random "noise" (not related to the stimulus) of the EEG. In addition, many other electrical sources from the head and body (eye movements, electrocardiogram, and muscle movements) and electromagnetic sources coming from cabling within the lab will also get picked up by the electrodes. This is also considered to be noise artifact. The signal is thus very difficult to isolate because it is embedded in the much larger background noise. Signal averaging techniques, originally developed in the 1950s (Dawson, 1954) can however be used to extract the ERP signal from the background noise.

ERPs consist of a series of positive and negativegoing deflections or "components", reflecting different hypothetical stages of cognitive processing. These components can be classified as being either "exogenous" when the ERP waveform is mainly affected by the physical properties of the stimulus, or "endogenous" when they are mainly affected by the more psychological properties such as decision-making, memory search, and meaningfulness (Sutton, Braren, Zubin, \& John, 1965). For this reason, many researchers use the terms, sensory and cognitive evoked potentials, to refer to the exogenous and endogenous ERPs, respectively. There has also been a recent tendency to consider ERPs to be only endogenous, although this was not the original definition (Vaughan, 1969). In general, ERPs that mainly reflect sensory processing occur early in processing while those that reflect mainly psychological events occur later. Some researchers thus classify ERPs according to their time (or latency) of occurrence as early (or "fast"), middle and late (or "slow") components.

\section{Stimulus Presentation}

Purely exogenous ERPs are affected only by the 
physical characteristics of the stimulus while purely endogenous ERPs are not affected by the physical features. They are however affected by factors such as the psychological relevance of the stimulus to the subject. For example, the subject's own name will elicit a large ERP, particularly for later components, not because of the physical features of the stimulus but because of the relevance of the stimulus to the subject. Another person's name, unknown to the subject, will elicit a very different ERP (Perrin et al., 2005).

Manipulation of the physical features of a stimulus should affect the exogenous but not the endogenous components of the ERP. In actual practice, distinguishing between the two types of components can be difficult. As an example, a subject is presented with an oddball paradigm in which they hear a frequently occurring "standard" $80 \mathrm{~dB}$ SPL $1000 \mathrm{~Hz}$ auditory tone. A tone is presented every $2 \mathrm{~s}$ (note that the physical characteristics of the stimulus must be described precisely because they will affect exogenous ERPs). At odd and unpredictable times, the intensity of the standard tone is increased to $90 \mathrm{~dB}$. The subject is asked to press a button upon detection of this rarely occurring increment "target". The standard tone will elicit a negativecomponent occurring at about $100 \mathrm{~ms}$ after stimulus presentation. This is called "N1". The target will also elicit an N1, but it will be larger in amplitude. Why would N1 be larger to the target? The most obvious explanation is that the target is physically different from the standard. It is more intense. N1 might vary directly in amplitude with the intensity of the auditory stimulus. In this case, N1 might be considered to be an exogenous ERP because it was altered by the manipulation of a physical feature. But in order for the subject to distinguish the target from the standard, they presumably will need to initially extract the features of the standard, store these features in working (or short-term) memory, and subsequently upon presentation of the target, compare its features to those that exist in memory for the standard. Memory comparison is a psychological process and thus perhaps the larger $\mathrm{N} 1$ reflects comparison of the features of the presently-occurring target to those that exist in memory for the standard. The detection of the target will also elicit a positive component occurring at about 300 ms, often labelled as "P3" (or "P300"). A P3 will not be apparent following presentation of the standard. P3 is often considered to be a classic endogenous component, related to the extent of the updating of working memory. How could the researcher resolve the problem of whether $\mathrm{N} 1$ and $\mathrm{P} 3$ are exogenous or endogenous? A simple way is to switch the standard and the target. What was formerly the standard stimulus (the $80 \mathrm{~dB}$ tone) is now the target and what was formerly the target stimulus (the $90 \mathrm{~dB}$ tone) is now the standard. If $\mathrm{N} 1$ varies only because of the physical features of the stimulus (its intensity in this example), it should be larger for the now more intense standard than the less intense target. On the other hand, if its amplitude reflects an endogenous process, such as a memory comparison process, then it should now be larger for the lower intensity target than the higher intensity standard. In actual fact, the N1 will still be larger to the higher intensity stimulus even though it is no longer a target. The $\mathrm{N} 1$ is thus largely exogenous in nature. Similarly, if P3 is an exogenous process (it occurred previously because the rare stimulus was more intense), then it should now also be larger to the more intense stimulus, in this case, the frequently occurring standard. In actual fact, the P3 will be larger to the infrequently presented target, regardless of its physical intensity. In other words, the occurrence of the P3 appears to almost exclusively reflect endogenous processing.

Even though the researcher might be mainly concerned about cognitive and psychological processing, care must be taken to ensure that the ERP component has not been affected by an incidental manipulation of physical features. At the same time, what the subject "is doing" (i.e. the subjects "task") is obviously critical if the ERP is affected by cognitive processing. Thus, the researcher must precisely describe (1) the physical characteristics of the stimuli and how often they are presented and (2) what is expected of the subject during the experimental task. The subject is often asked to signal their decision by button pressing, thus allowing the experimenter access to their cognitive (or "mental") state. In the example above, if the subject fails to detect the target, a P3 is not elicited although the exogenous $\mathrm{N} 1$ is. But, had the subject been asked to ignore the stimuli and simply relax, how would the experimenter have known what the subject was doing? Perhaps the subject did, in fact, ignore (i.e., not attend to) the stimuli and hence a P3 would not be elicited by the target. It is however also possible that the subject did attend to the stimuli and, in this case, a P3 would be elicited by the target. The mental state of a subject is essentially private but the researcher must design their experiment in a manner that allows access to it. 


\section{Recording of Performance Measures}

In the majority of ERP recording systems, two computers are used. One, the stimulus presentation computer, controls the presentation of the stimuli and the monitoring of subject responses. The second computer collects the EEG data. Both commercial software, such as E-Prime, Presentation, Psyscope, and open source software, such as PsychToolbox (within MatLab), or PsychoPy can be used for stimulus/response control and management (what stimulus is presented, when it is presented, and what the subject is asked to do). In general, the stimulus presentation and EEG data collection computers function independently and thus need to be synchronized. The EEG computer must know exactly when the stimulus is being presented and when a response has been made. A "trigger" being sent from a port on the stimulus presentation computer to a port on the EEG computer is often used for this purpose. In order to ensure at least millisecond precision for the analysis of the ERPs, the trigger must be sent out at precisely the time of stimulus presentation. The onus is on the researcher to ensure that an actual stimulus was presented exactly at the time the trigger is sent. In reality, there are often delays between the time the trigger is sent and the time a stimulus is actually presented. To verify the precision of visual stimulus presentation, light sensors can be used to detect the onset of the stimulus on the monitor. These sensors typically operate by detecting a change in the contrast and can be interfaced to the EEG electrode input box. It is also possible to employ an auditory sensor (a microphone) attached to the headphones used for auditory stimulus presentation. The auditory signal, in this case, can also be connected to the electrode input box. It is possible to connect the "line out" from the auditory generator to the electrode input box. In the case of both the visual and auditory stimuli, the output of the actual stimulus can be displayed on an EEG channel and then compared to the time of onset of the trigger (time 0 ). These should occur at the same time.

Most stimuli are now computer-generated. The sound chip in most computers does not have the quality required for precise scientific studies. A sound card should be added to the computer, if possible. The intensity of the sound needs to be precisely calibrated with a sound pressure level (SPL) meter. Different headphones have different impedances. Thus, a sound that measures $70 \mathrm{~dB}$ SPL using one headphone may not measure 70 dB SPL with another headphone. Importantly, headphones also have different frequency response characteristics. Thus, a $1000 \mathrm{~Hz}$ tone may measure $70 \mathrm{~dB}$ and also $70 \mathrm{~dB}$ at $2000 \mathrm{~Hz}$ but may measure $65 \mathrm{~dB}$ at $2000 \mathrm{~Hz}$ with a different pair of headphones. It is thus critical to examine the frequency response curve of the headphones. Manufacturers and websites may provide this information. As an example, the HeadRoom Inc web site, www.headphone.com, provides frequency response curves for various commercial headphones. These are used in Figure 1 to compare the frequency response curve of an Etymotic ER-4P insert earphone (often used in ERP research), and Sony MDR-V6, and Sennheiser HD280 headphones. A 90 SPL $1000 \mathrm{~Hz}$ signal is presented through each and the intensity of the signal is adjusted to be equal for all headphones at this frequency (i.e., the $90 \mathrm{~dB} 1000 \mathrm{~Hz}$ tone measures $90 \mathrm{~dB}$ for the three headphones). Most ERP studies present auditory stimuli ranging in frequency from about 500 to $2000 \mathrm{~Hz}$, but can be as low as $250 \mathrm{~Hz}$ and as high as $4000 \mathrm{~Hz}$. In Fig.1, the frequency response curves between 200 and $4000 \mathrm{~Hz}$ are highlighted. Between 200 and $2000 \mathrm{~Hz}$, the frequency response of the three headphones is relatively flat although the intensity of $1500-2000 \mathrm{~Hz}$ pure tones will be attenuated by 2 to $3 \mathrm{~dB}$ with the Sennheiser headphones. At 3000 to $4000 \mathrm{~Hz}$, the ER-4P and Sennheiser's intensity may fall as much as 3 to 6 $\mathrm{dB}$. This is not incidental. If the N1 ERP component is recorded to 1000 and $4000 \mathrm{~Hz}$ tones using the ER-4P and Sennheiser headphones, N1 will be reduced in amplitude for the $4000 \mathrm{~Hz}$ tone. Is this because of a difference in processing of the higher frequency (i.e., N1 is lower in amplitude for higher frequencies even if the headphone faithfully presents a $4000 \mathrm{~Hz}$ frequency) or is it because of the fact that the intensity is lower for the $4000 \mathrm{~Hz}$ stimulus when presented through these headphones? As may be observed, the three headphones (and almost all others) perform poorly above $5000 \mathrm{~Hz}$. Other factors such as subject comfort and the ability to isolate/attenuate ambient noise also need to be considered. In the examples above, the Sony headphones provide an excellent frequency response between 200 and $4000 \mathrm{~Hz}$ and are comfortable, but at a cost. Their ability to isolate ambient noise is poorer than, for example, the Sennheiser headphones. The tightly fitting in-ear ER-4P earphones offer excellent noise isolation but also at a cost; many subjects find them to be uncomfortable.

Visual stimuli are usually presented on a monitor. 


\section{Headphone Frequency Response Curve}

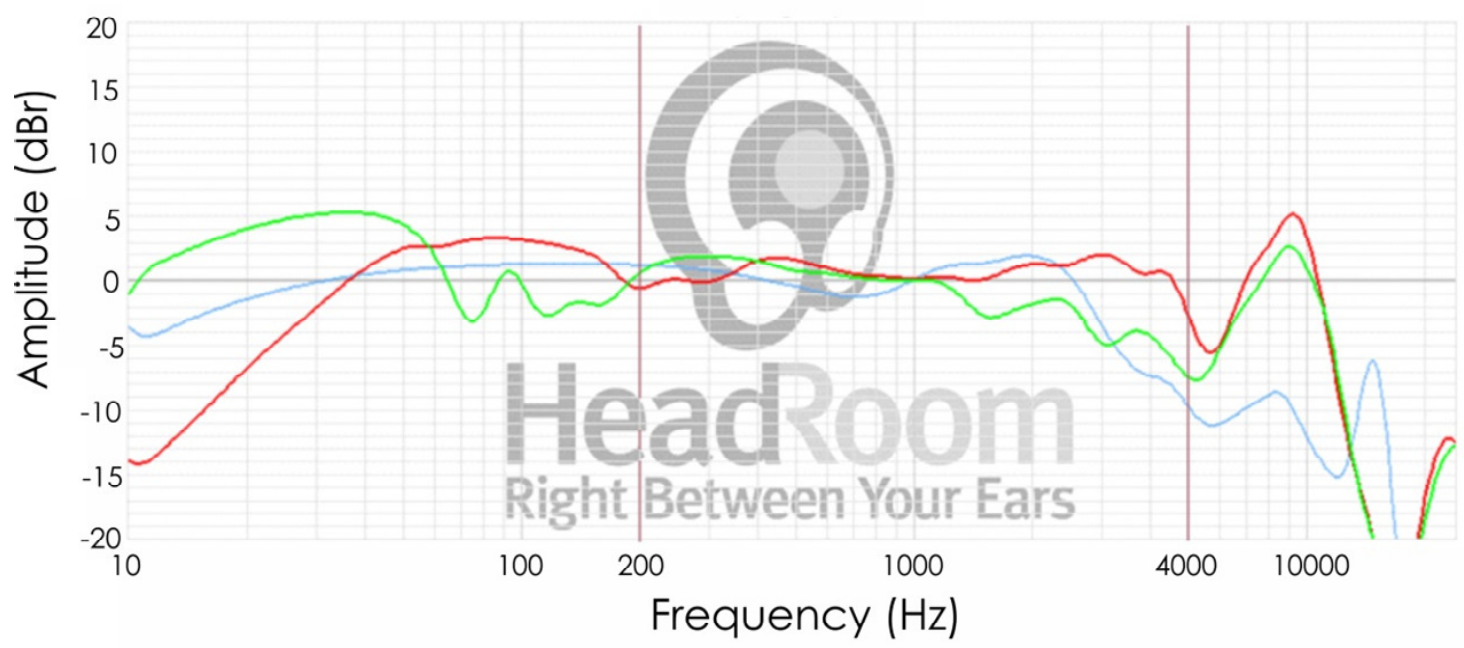

— Etymotic ER-4P — Sony MDR-V6 —Sennheiser HD280

Figure 1 - The frequency response curves of three different headphones are compared. The Etymotic earphone inserts are commonly used in ERP research. The choice of headphones is not incidental. A $1000 \mathrm{~Hz}$ tone was passed through each headphone and its intensity $(90 \mathrm{~dB})$ equated for each of the headphones. Thus, the response is identical for all three headphones at $1000 \mathrm{~Hz}$. From about 500 to $1000 \mathrm{~Hz}$, the intensity that is presented by each of the three headphones is about the same. From $1000-2000 \mathrm{~Hz}$, the intensity of the tone is attenuated somewhat when presented through the Sennheiser headphones. At about $3000 \mathrm{~Hz}$, the intensity is attenuated by more than $5 \mathrm{~dB}$ for both the Sennheiser and ER-4P earphones but faithfully reproduced by the Sony headphones. As may be observed, the three headphones perform poorly above $4000 \mathrm{~Hz}$.This image is courtesy of www.headphone.com.

The researcher should, however, be aware that most monitors are poorly calibrated in terms of their colour spectrum, intensity and contrast. Thus, if it is essential that a pure "red" stimulus be presented, on a pure "white" background, then the researcher would need to measure the wavelength of the stimuli. Calibrators exist for precisely this purpose. Intensity of the light wave may also be critical. Nevertheless, in many cognitive studies, approximation is sufficient. Thus, if a researcher is studying selective attention and asking subjects to detect red triangles among red rectangles, green triangles, and green rectangles, there is obviously much less need to be extremely precise. The red stimulus, even if it not a pure red, will still be much redder than the green stimulus. What is perhaps more important in visual research is the fact that there may be a very long delay between the time a trigger is sent to the EEG computer and when the stimulus is actually presented on the monitor. This can be as long as $40 \mathrm{~ms}$ especially for some LCD monitors, depending on the software that is used. This is much less of a concern for older CRT monitors. The precision of timing can be determined by using a simple sensor that detects change in contrast on the monitor. The output of this device can then be sent as an input to an EEG channel allowing a comparison of when a trigger was sent to when the stimulus was actually presented.

Subjects normally signal a response by button pressing. Often, a keyboard or mouse click is used. Neither keyboard nor mouse buttons provide precise 1 ms timing. Researchers who need 1 ms precision often employ external response devices. As an example, if reaction times (RTs) in an experiment to one type of stimulus are expected to be $10 \mathrm{~ms}$ faster than to another type of stimulus, it is unlikely that a keyboard or mouse will provide this type of precision. On the other hand, if the experimental effect is expected to be very large (e.g., more than a $100 \mathrm{~ms}$ difference), then special hardware will not be required. Some researchers have also noted that keyboards and mice can reveal even small changes in RT provided the data are averaged over a sufficiently large number of trials. Thus, the difference in the mean RTs will be precise even if single trial RTs are not (Segalowitz \& Graves, 1990; Shimizu, 2002), but again, the cost will be that many trials will be needed. 


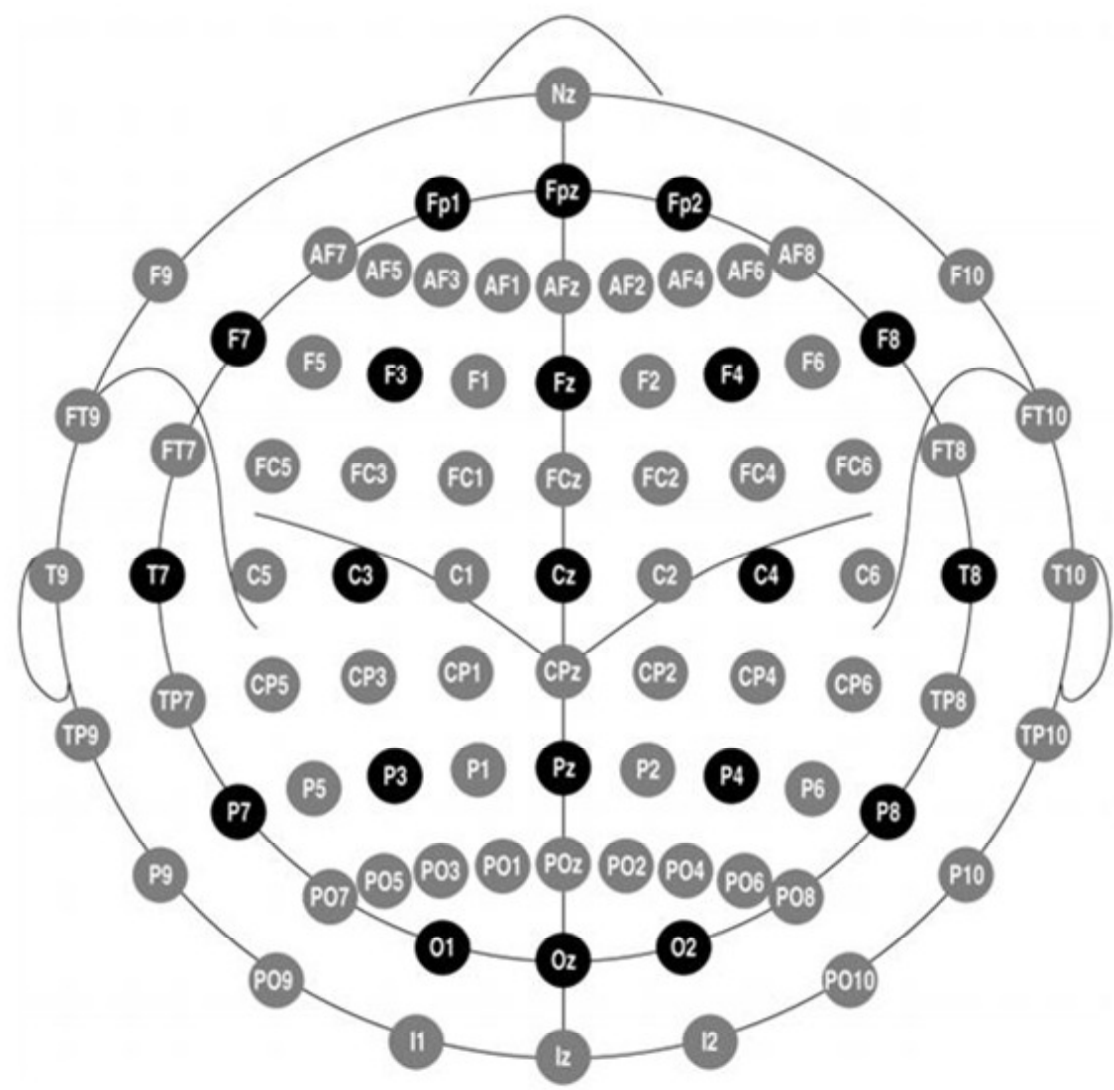

Figure 2 - Standardized system for the placement of electrodes on the scalp. The head is divided into proportional distances from prominent skull landmarks, the nasion (Nz), the inion (Iz) and the middle of the two ears (preauricular points). Electrodes used traditionally in the 10-20 system are darkened. Electrodes between these positions and more lateral and inferior that are used in the 10-10 system are shaded in grey.

\section{EEG Acquisition}

Small amplitude ERPs are embedded in the ongoing larger amplitude EEG. The EEG reflects the summed activity of thousands of postsynaptic potentials (the voltage that arises when a neurotransmitter binds to a postsynaptic site). EEG is almost always recorded from scalp electrodes in human subjects but the delay between the actual source signal within the brain and that recorded on the scalp is minimal. The intra-cranial neuronal population creates a dipole, resulting in positive and negative electrical charges separated by a small distance. Brain matter, the skull and skin do however cause large resistance to the flow of the dipole and the activity recorded on the scalp and will thus reduce the amplitude of the signal recorded at the scalp. As will be discussed throughout this article, it is critical to minimize the amount of resistance on the scalp in order to record good quality data.

Electrode Montage. In order to interpret ERPs, the EEG must be recorded simultaneously from multiple electrode sites. ERPs consist of a series of negative- and positive going components. One way to distinguish the various components is on the basis of their scalp distribution (where the response is large or small). Some components may, for example, be largest over frontal areas of the scalp. Other components might be largest over perhaps the right parietal areas. The two components are thus distinguished on the basis of different scalp distributions. Obviously, the scalp distribution of a component cannot be determined without recording the EEG from many different electrode sites. If the intra-cranial (within the brain) sources of the scalp-recorded ERP are already known, only a few electrodes will need to be placed. When only a few electrodes are employed, these can be attached to the scalp using a paste/cement. A gel is used to conduct the signal from the scalp to the electrode. While we do understand the sources of many of the exogenous components, generally little is known about the more 
endogenous components. For this reason, the endogenous components are often recorded using 32 to 256 electrode placements. For the placement of a multichannel montage, electrode caps with the electrodes embedded within the cap are usually preferred.

The placement of these electrodes on the scalp is called the "montage". A standardized placement and naming system, the 10-20 system, was developed in the early days of EEG recordings (Jasper, 1958). The head is divided into proportional distances from prominent skull landmarks (e.g. the nasion and inion) and the middle of the two ears (pre-auricular points) to provide adequate coverage of all regions of the brain. The label 10-20 refers to proportional distances where electrodes are located relative to these landmark points (Figure 2). Thus, electrode $\mathrm{Oz}$ is located $10 \%$ of the distance from the inion to the nasion. Electrode $\mathrm{Pz}$ is located $30 \%$ of the distance from the inion to the nasion (i.e., it is $20 \%$ anterior to $\mathrm{Oz}$ ). Electrode letters correspond to the major regions of the brain (FP = Frontal Polar, $\mathrm{F}=$ Frontal, $\mathrm{C}=$ Central, $\mathrm{P}=$ Parietal, $\mathrm{T}=$ Temporal, $\mathrm{O}=$ Occipital) followed by a number indicating the distance from the midline (designated by " $\mathrm{z}$ ") and whether the electrode is over the left (odd numbers) or right (even numbers) hemisphere. Thus, electrode placement F7 is located over the left frontal region. It is more lateral (or farther) from the midline (electrode $\mathrm{Fz}$ ) than electrode $\mathrm{F} 3$. The original 10-20 system did not allow for electrodes that were closely spaced and was thus subsequently modified (American Electroencephalographic Society, 1991) permitting intermediate positions. This is often called the 10-10 system. Thus, electrode POz is located $10 \%$ of the inionnasion distance anterior from $\mathrm{Oz}$ (or more simply, it is halfway between $\mathrm{Oz}$ and $\mathrm{Pz}$ ). Non-standard placements can also be used and these placements can be measured using modern 3-dimensional scanners. The use of scanning techniques to measure electrode placement has the advantage that the electrodes can be more or less placed arbitrarily on the scalp. In addition, the digitization of the electrode location permits the determination of the shape of the skull.

The Reference. All electrical signals are recorded from two points. In a referential montage, each of the EEG channels record the difference in the electrical activity between the scalp electrode and a common "reference" electrode. Ideally, the reference electrode should be inactive. A recording from a truly neutral reference electrode would be a straight line measuring $0 \mu \mathrm{V}$. It would not record any electrical activity from the brain. In reality, no reference site is truly inactive. The electrical activity of the brain spreads to all areas of the scalp including the reference site. Many articles have now described the effects of different reference sites. Common references include the mastoid, the ear lobes, or the tip of the nose, all of which pick up minimal brain electrical activity. Some researchers employ an average reference (the average of electrical activity from all electrode sites). The average reference does approximate a true zero voltage. However, in order to operate properly, electrodes must be placed not only on the upper and inferior, lateral surface of the scalp but also the face and neck regions. For this reason, it is not often used. Mastoid and earlobe references pick up some activity from both the auditory and the visual regions. Another problem with the choice of a reference using only a single placement, on for example, the left or right mastoid or ear lobe, is that if the cognitive activity is lateralized to one hemisphere of the brain, asymmetry of processing might either be canceled or enhanced by the reference. Had a truly neutral reference been employed, the lateralization might not have been observed. Many researchers thus opt to use a linked mastoid/ear lobe reference, averagingthe activity from both sites. Unfortunately, this solution will also be problematic if the impedance (described below) of the two reference sites varies over the course of the recording session, the virtual location of the reference would then be unknown. Some researchers use a midline scalp location for the reference. For example, a midline frontal (Fz) reference is often used for visual ERPs. This overcomes the problem of lateralized reference on one side of the scalp, when the ERP is thought to be lateralized. Scalp sites are however often very active. Some researchers thus prefer the tip of the nose as a reference. Even the tip of the nose is not truly inactive, although it does have the advantage that it is not lateralized. A major advantage of modern digital data acquisition systems is that the EEG can later be rereferenced to a different site, or even the linked mastoid/ear lobe reference, assuming of course that the EEG has also been recorded from the site(s) that will later become the reference (see Luck, 2014 for a review on referencing). Figure 3 illustrates an averaged ERP waveform elicited by a moderate intensity auditory stimulus. The effects of different references, the nose (left portion) the right mastoid (middle portion; M2), and the average of all electrode sites (right portion), are apparent. The EEG was recorded from 64 different 


\section{Reference Site}
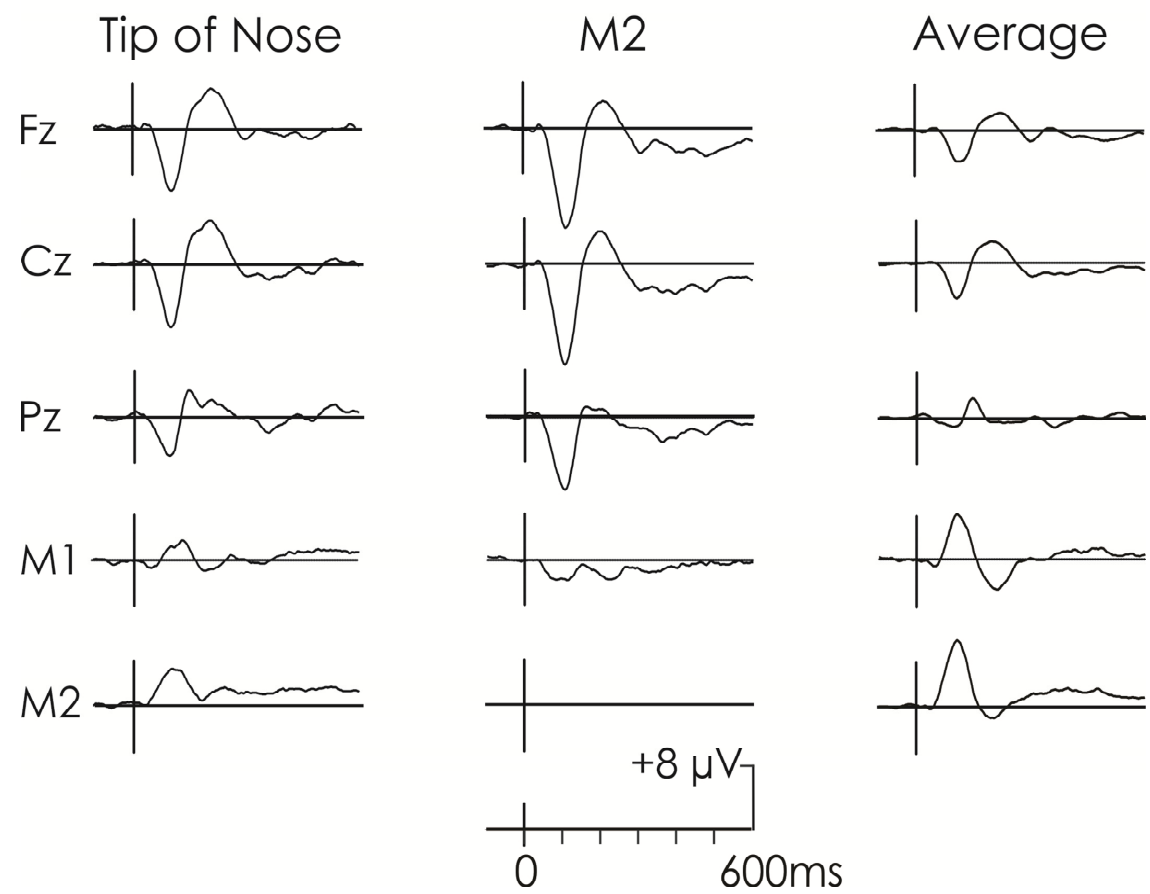

Figure 3 - The effects of choice of reference site. The activity of all scalp sites is usually compared to that at a neutral, inactive reference site. In reality, no reference site is truly neutral. All reference sites will record some EEG activity. In this example, an auditory ERP was elicited by a moderate intensity, $1000 \mathrm{~Hz}$ tone presented every $2 \mathrm{~s}$. In the left column, the tip of the nosewas chose as a reference. A large downward (negative) deflection is apparent at the $\mathrm{Fz}$ and $\mathrm{Cz}$ sites. The mastoid is often used as a reference site in many ERP studies. Note however that is certainly not inactive when auditory stimuli are presented. A large positive deflection is apparent at the mastoids when the tip of the nose is used as a reference. This inversion of polarity of $\mathrm{N} 1$ at the mastoids can be explained by the vertical orientation of a dipole source located in the nearby temporal lobe. In the middle portion of the Figure, the data were re-referenced to the right mastoid (M2). Now, the N1 is much large at both Fz and Pz when the positivity at the right mastoid is subtracted from the negativity recorded at these scalp sites. In the right column, the data were again rereferenced but an averaged reference (the average of activity at all electrode sites) was used. A clear inversion of polarity is again visible at the mastoids.

electrode sites including inferior sites, thus allowing the use of an averaged reference. A large downward deflection apparent at $\mathrm{Fz}$ and $\mathrm{Cz}$ can be observed to occur at about $100 \mathrm{~ms}$ after stimulus onset. When a nose reference is used, $\mathrm{N} 1$ is recorded as a negative wave at $\mathrm{Fz}$ (measuring about $-5.1 \mu \mathrm{V}$ ) and $\mathrm{Cz}$ but as a positive wave at the mastoids, M1 and M2 (measuring about $+3.0 \mu \mathrm{V}$ at M2). It is thought that the intracranial (within the brain) dipole sources of N1 are located within the auditory cortex of the temporal lobe. The dipole is oriented such that one pole "points" to the frontal region (thus explaining the negativity over frontal region) but the other pole points to the mastoid region and hence $\mathrm{N} 1$ is recorded with an opposite (or positive) polarity. The mastoids are therefore not inactive for auditory stimuli. Many researchers do employ a mastoid reference (centre portion). When the data were referenced to the right mastoid, the amplitude of $\mathrm{N} 1$ at $\mathrm{Fz}$ and $\mathrm{Cz}$ now becomes larger, measuring $-8.1 \mu \mathrm{V}$ (reflecting the difference in activity between $\mathrm{Fz}$ and $\mathrm{M} 2=-5.1-+3.0 \mu \mathrm{V}$ ). The data were also referenced to an average reference. Now, N1 measures $-2.7 \mu \mathrm{V}$ whereas it measures $+5.4 \mu \mathrm{V}$ at the mastoids. The choice of the reference may widely affect the data.

Impedance. A good electrical signal between the scalp and the electrodes is essential in the recording of high quality data. The quality of this connection can be quantified by measuring the impedance between the scalp and electrodes. Impedance is the tendency of a particular material to impede the flow of an alternating electrical current (the EEG in this case). The scalp is 
covered by a layer of dead skin cells and oils and has an impedance of about 50 or more kOhms. Minimizing the electrode impedance will result in a cleaner signal (see Teplan, 2002 for a detailed account). This impedance should be below $5 \mathrm{kOhms}$ for so-called passive electrode systems. It can be much higher (20-50 $\mathrm{kOhms)}$ for active systems. To reduce impedance below $5 \mathrm{kOhms} \mathrm{requires} \mathrm{the} \mathrm{scalp} \mathrm{to} \mathrm{be} \mathrm{rubbed} \mathrm{vigorously} \mathrm{and}$ often abraded using a blunt needle to break the initial layer of skin. Such vigorous rubbing and abrading is not required with active electrode systems.

Active vs. Passive Electrode Systems. EEG is recorded from metal electrodes attached to the scalp. Many different types of electrodes exist, each with different characteristics. Most metals used in the electrodes act as capacitors and store a charge after the electrode is removed. Thus, when it is placed on the next subject, an electrical charge may be recorded not because of the electrical activity from the brain of that subject but because of the stored charge on the electrode. For this reason, silver-silver chloride electrodes are often used in modern systems because of their non-polarizing properties, thus not storing a charge (Kutas, 1997; Rösler, Heil, \& Hennighausen, 1995). These electrodes are however very expensive relative to other types of electrodes.

Brain activity rarely exceeds $100 \mu \mathrm{V}$. An amplifier is required to make this signal visible.Most EEG systems use "passive" electrode amplifier systems. The EEG, once picked up by the electrodes, is relayed to the EEG amplifier usually via a cable measuring about 1-2 metres in length. Unfortunately, the cable connecting the electrodes to the amplifier also acts as an antenna and can pick up other electro-magnetic signals in the testing room. The amplifier, therefore, also amplifies artifactual noise and this will summate with the true brain signal. The EEG signal is thus a combination of a true EEG signal and noise. Electrical activity is picked up from the point of least resistance. This is why electrode impedance must be significantly reduced (with passive systems). "Active" electrode systems embed a small pre-amplifier within each electrode casing. Therefore, the distance required for the signal to travel from the electrode to the amplifier is very short; hence, the possibility of picking up additional noise is minimized. They also overcome the need for vigorous abrasion of the scalp and thus are often the electrodes of choice with infants, young children and patient populations. Active electrode systems are nevertheless quite expensive and are also quite large because of the attached amplifier. They may not be appropriate in all situations, for example, during sleep studies when the smaller and flatter passive electrodes are less intrusive.

\section{Conclusion}

Modern technological innovations now permit the recording of the EEG from as many as 256 different channels. Low cost but highly sophisticated hardware and general purpose software now enable many researchers to record real-time mental processing using well-established cognitive paradigms. In this article, we have highlighted some of the techniques required for the presentation of stimuli and the acquisition of good quality data. In spite of the power of new techniques, due diligence must still be afforded to the careful and precise presentation of the stimulus and a monitoring of the behavior/performance of the subject. Poor research design will not result in good cognitive neuroscience. While the EEG recording capabilities have expanded enormously over the past decade, the researcher must always be aware that the electrical activity that is recorded is a summation of a true brain signal and background noise. Part II of this article will describe methods that can be used to reduce this background noise activity and quantify the signal, but it is always best to reduce the noise as much as possible in original, real-time recording. "Prevention is better than cure" (Picton, 1995, p.20).

\section{References}

Dawson. G.D. (1954). A summation technique for the detection of small evoked potentials. Electroencephalography and Clinical Neurophysiology, 6, 6584.

Jasper, H.H. (1958). The ten-twenty electrode system of the International Federation. Electroencephalography and Clinical Neurophysiology, 10, 371-375.

Kutas, M. (1997). Views on how the electrical activity that the brain generates reflects the functions of different language structures. Psychophysiology, 34, 383-398.

Luck, S. J. (2014). An introduction to the event-related potential technique. MIT press.

Perrin, F., Maquet, P., Peigneux, P., Ruby, P., Degueldre, C., Balteau, E., ... \& Laureys, S. (2005). Neural mechanisms involved in the detection of our first name: a combined ERPs and PET study. Neuropsychologia, 43, 12-19.

Picton, T.W., Lins, O.G., \& Scherg, M. (1995). The recording and analysis of event-related potentials. 
The Handbook of Neuropsychology, 10, 3-73.

Rösler, F., Heil, M., \& Hennighausen, E. (1995). Distinct cortical activation patterns during long-term memory retrieval of verbal, spatial, and color information. Journal of Cognitive Neuroscience, 7, 51-65.

Segalowitz, S. J., \& Graves, R. E. (1990). Suitability of the IBM XT, AT, and PS/2 keyboard, mouse, and game port as response devices in reaction time paradigms. Behavior Research Methods, Instruments, \& Computers, 22, 283-289.

Sharbrough, F., Chatrian, G. E., Lesser, R. P., Lüders, H., Nuwer, M., \& Picton, T. W. (1991). American Electroencephalographic Society guidelines for standard electrode position nomenclature. Clinical Neurophysiology, 8, 200-202.
Shimizu, H. (2002). Measuring keyboard response delays by comparing keyboard and joystick inputs. Behavior Research Methods, Instruments, \& Computers, 34, 250-256.

Sutton, S., Braren, M., Zubin, J., \& John, E. R. (1965). Evoked-potential correlates of stimulus uncertainty. Science, 150, 1187-1188.

Teplan, M. (2002). Fundamentals of EEG measurement. Measurement Science Review, 2, 1-11.

Vaughan, H. G., Jr. (1969). The relationship of brain activity to scalp recordings of event-related potentials. In Donchin, E., and Lindsley, D. B. (eds.), Average Evoked Potentials, National Aeronautics and Space Administration, Washington, D.C., pp. 4594.

\section{Citation}

Tavakoli, P., \& Campbell, K. (2015). The Recording and Quantification of Event-Related Potentials: I. Stimulus Presentation and Data Acquisition. The Quantitative Methods for Psychology, 11(2), 89-97.

Copyright (C) 2015 Tavakol \& Campbell. This is an open-access article distributed under the terms of the Creative Commons Attribution License (CC BY). The use, distribution or reproduction in other forums is permitted, provided the original author(s) or licensor are credited and that the original publication in this journal is cited, in accordance with accepted academic practice. No use, distribution or reproduction is permitted which does not comply with these terms.

Received: 4/12/14 Accepted: 26/03/15 